\title{
DOWNLINK MBER BEAMFORMING TRANSMITTER BASED ON UPLINK MBER BEAMFORMING RECEIVER FOR TDD-SDMA MIMO SYSTEMS
}

\author{
Sheng Chen and Lie-Liang Yang \\ School of ECS, University of Southampton, Southampton SO17 1BJ, UK
}

\begin{abstract}
The downlink minimum bit error rate (MBER) transmit beamforming is directly derived based on the uplink MBER receive beamforming for time division duplex (TDD) space-division multiple-access (SDMA) multiple-input multiple-output systems, where the base station (BS) is equipped with multiple antennas to support multiple single-antenna mobile terminals (MTs). It is shown that the relationship between multiuser detection and multiuser transmission can still be applied for the rank-deficient system where the number of users supported is more than the number of transmit antennas available at the BS, if the MBER design is adopted. The proposed MBER transmit beamforming scheme is capable of achieving good performance for rank-deficient TDD-SDMA systems with the support of low-complexity and high power-efficient MTs, and its robustness to the downlink and uplink noise or channel mismatch is verified using simulation.
\end{abstract}

Index Terms - Minimum bit error rate, time division duplex, transmit beamforming, space-division multiple-access

\section{INTRODUCTION}

Motivated by the demand for increasing throughput in wireless communication, antenna array assisted spatial processing techniques $[1,2,3,4,5,6,7]$ have been developed in order to further improve the achievable spectral efficiency. In the uplink, the base station (BS) has the capacity to implement sophisticted receive beamforming $(\mathrm{RxB})$ schemes to separate multiple user signals transmitted by mobile terminals (MTs). This provides a practical means of realising multiuser detection (MUD) for the space-division multiple-access (SDMA). Traditionally, adaptive RxB is based on the minimum mean square error (MMSE) design [1, 4, 5, 8], which requires that the number of users supported is no more than the number of receive antenna elements. If this condition is not met, the system becomes rank-deficient. Recently, adaptive minimum bit error rate (MBER) RxB design has been proposed $[9,10,11,12]$, which outperforms the adaptive MMSE RxB, particularly in hostile rank-deficient systems.

In the downlink with non-cooperative MTs at the receive end, the mobile users are unable to perform sophisticated cooperative MUD. If the downlink's channel state information is known at the BS, the BS can carry out transmit preprocessing, leading to multiuser transmission (MUT). The assumption that the downlink channel impulse response is known at the BS is valid for time division duplex (TDD) systems due to the channel reciprocity. Many research effects have been made to discover the equivalent relationship between the MUD and MUT [13, 14, 15, 16, 17, 18]. Notably, Yang [19] has derived the exact equivalency between the MUD and MUT for TDD systems under the condition that the number of antennas at the BS is no less than the number of MTs supported. According to the results of [19], the MUT can be obtained directly from MUD. Since the BS has to implement MUD, it can readily implement MUT with no extra computational complexity cost. This is very attractive as this strategy enables the employment of low-complexity and high powerefficient MTs to achieve good downlink performance.

This work applies the results of [19] to rank-deficient TDDSDMA systems where the number of antennas at the BS is less than the number of MTs supported. Specifically, we implement the MBER TxB scheme directly based on the MBER $\mathrm{RxB}$ scheme. The simulation results obtained clearly show that Yang's results [19] can still be applied for rank-deficient systems, if the MBER design instead of the MMSE design is adopted, and the proposed MBER TxB design significantly outperforms the MMSE TxB design. The robustness of the proposed scheme is also investigated when the downlink and uplink noise powers or channels mismatch.

\section{MULTIUSER BEAMFORMING SYSTEM}

The TDD-SDMA system considered is depicted in Fig. 1, where the BS employs $L$ antennas to support $K$ single-antenna MTs. When the uplink is considered, the received signal vector $\mathbf{x}_{U}=\left[x_{U, 1} x_{U, 2} \cdots x_{U, L}\right]^{T}$ at the BS is given by

$$
\mathbf{x}_{U}=\mathbf{H} \mathbf{s}+\mathbf{n}_{U}=\sum_{k=1}^{K} \mathbf{h}_{k} s_{k}+\mathbf{n}_{U}
$$

where the $L \times K$ channel matrix is given by $\mathbf{H}=\left[\mathbf{h}_{1} \cdots \mathbf{h}_{K}\right]$, $\mathbf{h}_{k}=A_{k} \mathbf{g}_{k}$ with $A_{k}$ and $\mathbf{g}_{k}$ denoting the channel coefficient and the steering vector for the $k$ th user, respectively, $\mathbf{s}=\left[\begin{array}{llll}s_{1} & s_{2} & \cdots & s_{K}\end{array}\right]^{T}$ contains the $K$ data symbols transmitted by the $K$ MTs to the BS, and $\mathbf{n}_{U}$ denotes the uplink 


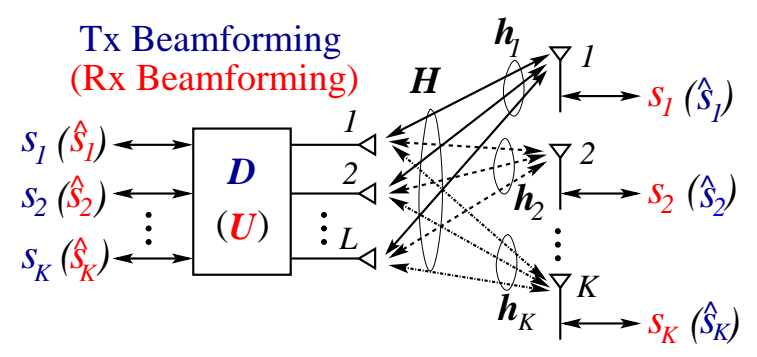

Fig. 1. Schematic diagram of the TDD-SDMA multiple-input multiple-output system employing transmit and receive beamforming at the base station (BS). The system employs $L$ antennas at the BS to support $K$ single-antenna mobile users.

channel additive white Gaussian noise (AWGN) vector with $E\left[\mathbf{n}_{U} \mathbf{n}_{U}^{H}\right]=2 \sigma_{U}^{2} \mathbf{I}_{L}$, and $\mathbf{I}_{L}$ represents the $L \times L$ identity matrix. Without the loss of generality, we assume the binary phase shift keying (BPSK) modulation. For modulation schemes with multiple bits per symbol, the minimum symbolerror-rate design [12] can be adopted. The MUD at the BS consists of a bank of receive beamformers

$$
y_{U, k}=\mathbf{u}_{k}^{H} \mathbf{x}_{U}, 1 \leq k \leq K,
$$

where $\mathbf{u}_{k}$ is the $\mathrm{RxB}$ weight vector for user $k$. The decision variable vector $\mathbf{y}_{U}=\left[\begin{array}{lll}y_{U, 1} & y_{U, 2} \cdots y_{U, K}\end{array}\right]^{T}$ for the $K$ transmitted symbols can be expressed as

$$
\mathbf{y}_{U}=\mathbf{U}^{H} \mathbf{x}_{U}=\mathbf{U}^{H} \mathbf{H} \mathbf{s}+\mathbf{U}^{H} \mathbf{n}_{U}
$$

with the $L \times K \mathrm{RxB}$ coefficient matrix expressed by

$$
\mathbf{U}=\left[\mathbf{u}_{1} \mathbf{u}_{2} \cdots \mathbf{u}_{K}\right] .
$$

The real part of $\mathbf{y}_{U}$ is a sufficient statistics for detecting $\mathbf{s}$.

The BS employs the TxB for the downlink transmission to $K \mathrm{MTs}$, with the $L \times K$ transmission preprocessing matrix

$$
\mathbf{D}=\left[\mathbf{d}_{1} \mathbf{d}_{2} \cdots \mathbf{d}_{K}\right],
$$

where $\mathbf{d}_{k}$ is the precoder's coefficient vector for preprocessing the symbol $s_{k}$ to be transmitted to the $k$ th MT. Note that we use the same notation $\mathbf{s}$ to represent the downlink symbol vector, without distinction from the uplink symbol vector for the purpose of notational simplification. Due to the reciprocity of the downlink and uplink channels, the received signal vector $\mathbf{y}_{D}=\left[\begin{array}{ll}y_{D, 1} & y_{D, 2} \cdots y_{D, K}\end{array}\right]^{T}$, received by the $K$ MTs, is expressed as

$$
\mathbf{y}_{D}=\mathbf{H}^{T} \mathbf{D} \mathbf{s}+\mathbf{n}_{D}
$$

where $\mathbf{n}_{D}$ is the downlink AWGN vector with $E\left[\mathbf{n}_{D} \mathbf{n}_{D}^{H}\right]=$ $2 \sigma_{D}^{2} \mathbf{I}_{L}$. Note that the real part of the decision variable $y_{D, k}$ is used by the $k$ th MT for detecting the symbol $s_{k}$ transmitted from the BS to the $k$ th MT.

Under the condition $L \geq K$, there exists an exact equivalency between the TxB preprocessing matrix $\mathbf{D}$ and the $\mathrm{RxB}$ weight matrix $\mathbf{U}$ expressed by [19]

$$
\mathbf{D}=\mathbf{U}^{*} \Lambda
$$

where $\boldsymbol{\Lambda}=\operatorname{diag}\left\{\lambda_{1}, \lambda_{2}, \cdots, \lambda_{K}\right\}$ is for achieving the transmit power constraint. The exact relationship (7) is valid for $\sigma_{D}^{2}=\sigma_{U}^{2}$. A simple scheme to implement the transmit power constraint is to set $\lambda_{k}=1 /\left\|\mathbf{u}_{k}\right\|$, for $1 \leq k \leq K$. The relationship (7) is easy to understand. Under the condition of $L \geq K, \mathbf{U}^{H} \mathbf{H}$ of (3) and $\mathbf{H}^{T} \mathbf{D}$ of (6) have the same full rank and the same statistic properties, given (7).

\section{MBER RECEIVE AND TRANSMIT BEAMFORMING}

For rank-deficient systems, $L<K$, and $\mathbf{U}^{H} \mathbf{H}$ and $\mathbf{H}^{T} \mathbf{D}$ no longer have the full rank. Indeed, both the MMSE RxB and MMSE TxB turn out to be deficient in this case. However, the MBER RxB scheme $[9,10]$ has been shown to consistently outperform the MMSE design and is capable of operating in rank-deficient systems where the number of MTs is more than the number of receive antennas at the BS. We therefore use (7) to derive the MBER TxB design directly from the MBER RxB solution. Although for the case of $L<K$ the equivalency between the MUD and MUT may no longer be exact, the relationship (7) can still be applied for the MBER design, as will be shown in the simulation study.

The bit error rate (BER) of detecting $s_{k}$ using the uplink $\mathrm{RxB}$ with the weight vector $\mathbf{u}_{k}$ can be shown to be $[9,10]$

$$
P_{R x, k}\left(\mathbf{u}_{k}\right)=\frac{1}{N_{s b}} \sum_{q=1}^{N_{s b}} Q\left(\frac{\operatorname{sgn}\left(s_{k}^{(q)}\right) \Re\left[\mathbf{u}_{k}^{H} \mathbf{H s}^{(q, k)}\right]}{\left\|\mathbf{u}_{k}\right\| \sigma_{U}}\right),
$$

where $Q(\bullet)$ is the usual Gaussian error function, $\Re[\bullet]$ denotes the real part, $N_{s b}=2^{K-1}$, $\mathbf{s}^{(q, k)}$ for $1 \leq q \leq N_{s b}$ are the equiprobable legitimate transmit symbol vectors given $s_{k}^{(q)}=$ +1 , and $s_{k}^{(q)}$ denotes the $k$ th element of $\mathbf{s}^{(q, k)}$. The MBER solution for $\mathbf{u}_{k}$ is then defined as

$$
\mathbf{u}_{\mathrm{MBER}, \mathrm{k}}=\arg \min _{\mathbf{u}_{k}} P_{R x, k}\left(\mathbf{u}_{k}\right) .
$$

The optimisation (9) can be solved using a gradient-based numerical optimisation algorithm [10]. Note that the BER is invariant to a positive scaling of $\mathbf{u}_{k}$, and one can always normalised the beamforming weight vector to a unit length which significantly reduces the computational complexity of optimisation. This is also useful for directly implementing the TxB design from the RxB design using the relationship (7), as the scaling matrix $\Lambda$ becomes the identity matrix in this case. Adaptive implementation of the MBER RxB can be achieved using the stochastic gradient-based algorithm known as the least bit error rate $[9,10]$.

With the precoder coefficient matrix $\mathbf{D}$, the BER of detecting $s_{k}$ by the $k$ th MT is for $1 \leq k \leq K$

$$
P_{T x, k}(\mathbf{D})=\frac{1}{N_{s}} \sum_{q=1}^{N_{s}} Q\left(\frac{\operatorname{sgn}\left(s_{k}^{(q)}\right) \Re\left[\mathbf{h}_{k}^{T} \mathbf{D} \mathbf{s}^{(q)}\right]}{\sigma_{D}}\right),
$$


where $N_{s}=2^{K}$ is the number of all the equiprobable legitimate transmit symbol vectors, $\mathbf{s}^{(q)}$ for $1 \leq q \leq N_{s}$, and $s_{k}^{(q)}$ denotes the $k$ th element of $\mathbf{s}^{(q)}$. The MBER TxB solution $\mathbf{D}_{\text {MBER }}$ can be obtained by minimising the average BER over all the $K$ users

$$
P_{T x}(\mathbf{D})=\frac{1}{K} \sum_{k=1}^{K} P_{T x, k}(\mathbf{D})
$$

subject to the certain transmit power constraint. The resulting constrained nonlinear optimisation problem for example can be solved using the iterative gradient-based optimisation algorithm known as the sequential quadratic programming (SQP) $[20,21]$. Instread of applying this computationally expensive SQP algorithm to find the MBER TxB solution, we can directly derive it according to the relation (7) as

$$
\mathbf{D}_{\text {MBER }}=\mathbf{U}_{\text {MBER }}^{*},
$$

with no computational cost at all. The validity of this approach will be verified in the simulation study.

\section{SIMULATION STUDY}

The BS employed a four-element linear antenna array with half-wavelength element spacing to support six BPSK users. The angles of arrival (departure) for the six users were $-2^{\circ}$, $-15^{\circ}, 10^{\circ},-30^{\circ}, 25^{\circ}$ and $36^{\circ}$, respectively, and the channel coefficients for the six users were $A_{k}=0.7071+j 0.7071$, $1 \leq k \leq 6$. For the uplink, Fig. 2 compares the average BER performance, defined as

$$
P_{R x}(\mathbf{U})=\frac{1}{K} \sum_{k=1}^{K} P_{R x, k}\left(\mathbf{u}_{k}\right),
$$

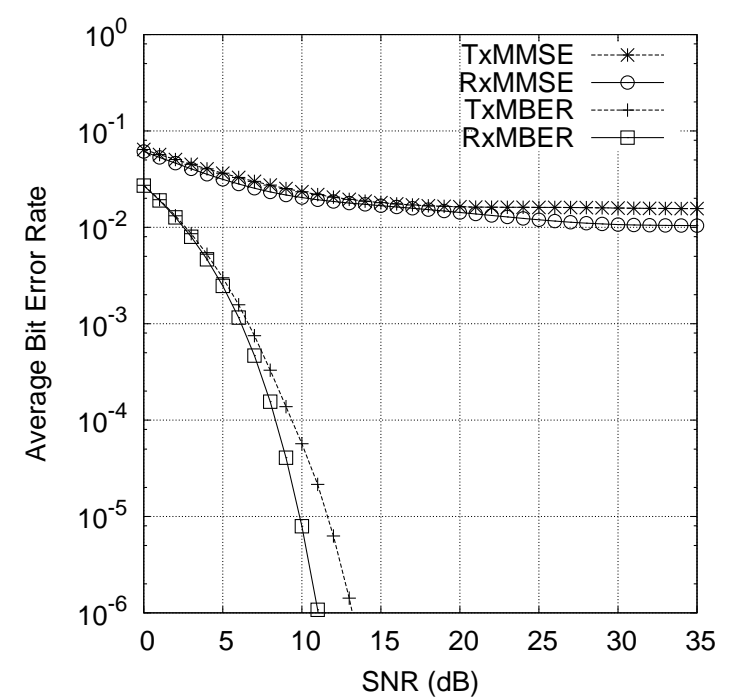

Fig. 2. Average BER performance comparison of the uplink $R x B$ and downlink TxB schemes with both the MMSE and MBER designs for the TDD-SDMA system which consists of a four-antenna BS to support six BPSK MTs.

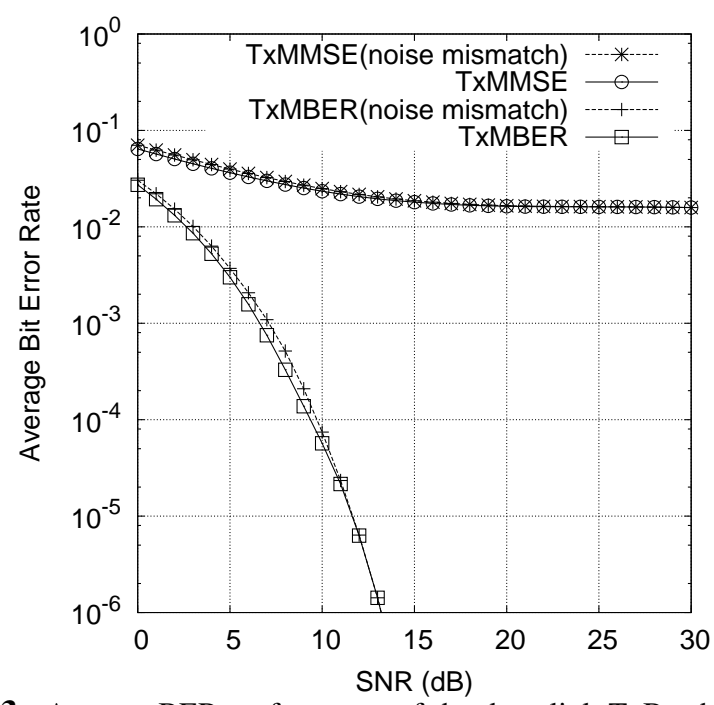

Fig. 3. Average BER performance of the downlink TxB schemes with both the MMSE and MBER designs for the TDD-SDMA system which consists of a four-antenna BS to support six BPSK MTs, when the downlink and uplink noise powers mismatch.

of the MBER and MMSE RxB designs. For this rank-deficient system, the MBER beamforming receiver significantly outperformed the MMSE beamforming receiver. We then implemented the downlink transmit beamforming directly based on the uplink receive beamforming based on the relation (7). The average BERs, as defined in (11), of both the MBER TxB and MMSE TxB so designed are also plotted in Fig. 2, in comparison with the average BERs of the MBER and MMSE RxB designs. It can be seen from Fig. 2 that the relation (7) can still be applied in the rank-deficient scenario.

The robustness of the proposed TxB design was next investigated when the downlink and uplink noise powers or channels mismatched. In the case of noise mismatch, the downlink noise power was $3 \mathrm{~dB}$ more than the uplink noise power. The average BERs of the MBER and MMSE TxB designs under this uplink/downlink noise power mismatching are plotted in Fig. 3, in comparison with the case of equal uplink and downlink noise powers. It can be seen that the $3 \mathrm{~dB}$ noise-power mismatching had little influence on performance. In the case of channel mismatch, the uplink channels were $A_{k}=0.7071+j 0.7071$ for $1 \leq k \leq 6$, but the downlink channels were $A_{k}=0.6+j 0.8$ for $1 \leq k \leq 6$. The average BER performance of the MBER and MMSE TxB designs under this uplink/downlink channel mismatching are plotted in Fig. 4, in comparison with the case of perfect channel state information. From Fig. 4, it can be seen that the MBER TxB design was not overly sensitive to the imperfect channel state information.

\section{CONCLUSIONS}

The downlink MBER transmit beamforming solution has been derived directly based on the uplink MBER receive beam- 


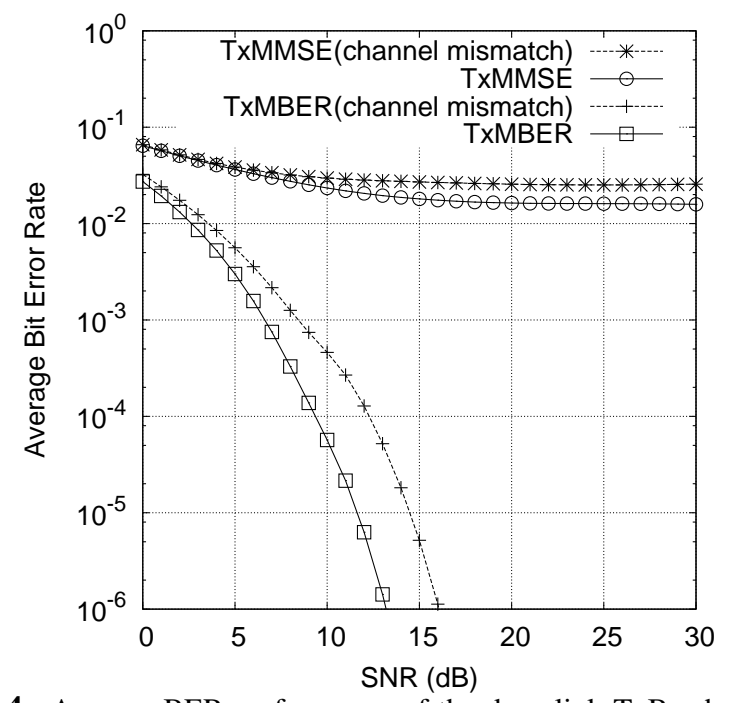

Fig. 4. Average BER performance of the downlink TxB schemes with both the MMSE and MBER designs for the TDD-SDMA system which consists of a four-antenna BS to support six BPSK MTs, when the downlink and uplink channels mismatch.

forming for TDD-SDMA multiple-input multiple-output systems, where the number of MTs supported is more than the number of transmit antennas available at the BS. The derivation has been based on the relationship between MUD and MUT which is for full-rank TDD-SDMA systems where the number of users supported is no more than the number of transmit antennas at the BS. It has been shown that this relation can still apply for rank-deficient systems, if the MBER design is adopted. The proposed MBER transmit beamforming scheme is capable of achieving good downlink performance with the support of low-complexity and high powerefficient MTs, and its robustness to the downlink and uplink noise or channel mismatch has been verified by simulation.

\section{REFERENCES}

[1] J. Litva and T. K. Y. Lo, Digital Beamforming in Wireless Communications, Artech House, London, 1996.

[2] J. H. Winters, "Smart antennas for wireless systems," IEEE Personal Communications, vol. 5, pp. 23-27, 1998.

[3] P. Vandenameele, L. van Der Perre and M. Engels, Space Division Multiple Access for Wireless Local Area Networks, Kluwer Academic Publishers, Boston, MA, 2001.

[4] J. S. Blogh and L. Hanzo, Third Generation Systems and Intelligent Wireless Networking - Smart Antennas and Adaptive Modulation, Wiley, Chichester, UK, 2002.

[5] A. Paulraj, R. Nabar and D. Gore, Introduction to Space-Time Wireless Communications, Cambridge University Press, Cambridge, UK, 2003.

[6] A. J. Paulraj, D. A. Gore, R. U. Nabar and H. Bölcskei, “An overview of MIMO communications - A key to gigabit wireless," Proc. IEEE, vol. 92, pp. 198-218, February 2004.
[7] D. Tse, and P. Viswanath, Fundamentals of Wireless Communication, Cambridge University Press, Cambridge, UK, 2005.

[8] D. N. C. Tse and S. V. Hanly, "Linear multiuser receivers: effective interference, effective bandwidth and user capacity," IEEE Trans. Information Theory, vol. 45, pp. 641-657, March 1999.

[9] S. Chen, L. Hanzo and N. N. Ahmad, "Adaptive minimum bit error rate beamforming assisted receiver for wireless communications," in Proc. ICASSP 2003, Hong Kong, China, April 6-10, 2003, vol. IV, pp. 640-643.

[10] S. Chen, N. N. Ahmad and L. Hanzo, "Adaptive minimum bit error rate beamforming," IEEE Trans. Wireless Communications, vol. 4, pp. 341-348, March 2005.

[11] S. Chen, L. Hanzo, N. N. Ahmad and A. Wolfgang, "Adaptive minimum bit error rate beamforming assisted receiver for QPSK wireless communication," Digital Signal Processing, vol. 15, pp. 545-567, November 2005.

[12] S. Chen, A. Livingstone, H.-Q. Du and L. Hanzo, "Adaptive minimum symbol error rate beamforming assisted detection for quadrature amplitude modulation," IEEE Trans. Wireless Communications, vol. 7, pp. 1140-1145, April 2008.

[13] D. N. C. Tse and P. Viswanath, "Downlink-uplink duality and effective bandwidths," in Proc. IEEE 2002 Int. Symp. Information Theory, Palais de Beaulieu, Switzerland, June 30-July 5, 2002, p. 52.

[14] M. Schubert and H. Boche, "Solution of the multiuser downlink beamforming problem with individual SINR constraints," IEEE Trans. Vehicular Technology, vol. 53, pp. 18-28, January 2004.

[15] M. Schubert and H. Boche, "Iterative multiuser uplink and downlink beamforming under SINR constraints," IEEE Trans. Signal Processing, vol. 53, pp. 2324-2334, July 2005.

[16] W. Yu, "Uplink-downlink duality via minimax duality," IEEE Trans. Information Theory, vol. 52, pp. 361-374, February 2006.

[17] A. M. Khachan, A. J. Tenenbaum and R. S. Adve, "Linear processing for the downlink in multiuser MIMO systems with multiple data streams," in Proc. ICC 2006, Istanbul, Turkey, June 11-15, 2006, vol. 9, pp. 4113-4118.

[18] S. Shi, M. Schubert and H. Boche, "Downlink MMSE transceiver optimization for multiuser MIMO systems: duality and sum-MSE minimization,” IEEE Trans. Signal Processing, vol. 55, pp. 5436-5446, November 2007.

[19] L.-L. Yang, "Design linear multiuser transmitters from linear multiuser receivers," in Proc. ICC 2007, Glasgow, Scotland, June 24-28, 2007, pp. 5258-5263.

[20] A. Hjørungnes and P. S. R. Diniz, "Minimum BER prefilter transform for communications systems with binary signaling and known FIR MIMO channel," IEEE Signal Processing Letters, vol. 12, pp. 234-237, March 2005.

[21] S. Tan, Minimum Error Rate Beamforming Transceivers, Ph.D. thesis, School of Electronics and Computer Science, University of Southampton, Southampton, UK, April 2008. 